\title{
Психомогия
}

\section{Педагогическая психомогия}

УДК 378.172

\section{Эмоциональное состояние студентов в период пандемии COVID-19 в странах с разными ограничительными мерами}

\section{The emotional state of students during the COVID-19 pandemic in countries with different restrictive measures}

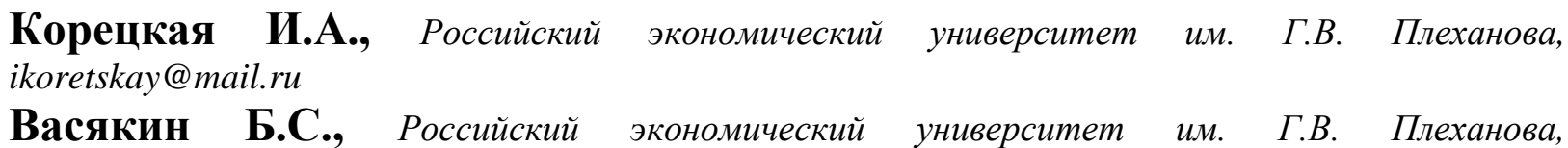
vasyakin.bs@rea.ru

Кая А.А., Российский экономический университет им. Г.В. Плеханова, alex2228@ list.ru

Koretskaya I., Plekhanov Russian University of Economics, ikoretskay@mail.ru

Vasyakin B., Plekhanov Russian University of Economics, vasyakin.bs@rea.ru

Kaya A., Plekhanov Russian University of Economics, alex2228@list.ru

DOI: $10.51379 / \mathrm{KPJ} .2021 .147 .3 .024$

Ключевые слова: эмочииональное состояние, COVID-19, HADS, тревога, депрессия.

Keywords: emotional state, COVID-19, HADS, anxiety, depression.

Аннотация. Пандемия COVID-19 во всех странах спровоцировала ограничения выхода из дома, перевод работы и обучения в онлайн форматы и соответственно изменилась организация учебного процесса. Тот факт, что данные ограничения повлияют на психику людей, не вызывал сомнения уже в начале пандемии, однако какие это будут изменения, и насколько глубоко они затронут людей, точных прогнозов никто дать не мог. Настоящее исследование направлено на определение уровней личной тревожности и депрессии студентов России и Туриии. Целью исследования было сравнить тревожность и депрессивность у турецких и российских студентов в период второй волны пандемии в конце 2020 года. Выбор стран был обусловлен разнылм государственным отношением к эпидемиологической обстановке в текущей ситуации.

В качестве методики исследования была выбрана госпитальная шкала тревоги и депрессии HADS (The Hospital Anxiety and Depression Scale), разработанная для первичного выявления депрессии и тревоги. Выбор методики был связан с тем, что она переведена на национальные языки России и Турции и соответствует всем психометрическим требованиям.

Пандемия COVID-19 повысила уровень тревожности, которая усиливается в стрессовых условиях жизни. Исходя из проведённого исследования, мы видим, что в стране, где более выраженная ситуация запрета, и введены новые, строгие нормы взаимодействия с окружающей средой, более выражено проявляется повышенный уровень тревоги и депрессии.

Abstract. The COVID-19 pandemic in all countries provoked restrictions on leaving home, transferring work and education to online formats and, accordingly, changing the organization of the educational process. There was no doubt at the beginning of the pandemic that these restrictions would affect people's psyche, but no one could predict exactly what these changes would be and how deeply they would affect people. The present study sought to determine levels of personal anxiety and depression among students in Russia and Turkey. The aim of the study was to compare anxiety and depression among Turkish and Russian students during the second wave of the pandemic at the end of 2020. The choice of countries was due to different governmental attitudes to the epidemiological situation in the current situation. 
The HADS (The Hospital Anxiety and Depression Scale), developed for the primary detection of depression and anxiety, was chosen as a research methodology. The choice of the technique was because it has been translated into the national languages of Russia and Turkey and meets all psychometric requirements.

The COVID-19 pandemic increased the level of anxiety, which increases in stressful living conditions. Based on the conducted research, we can see that in a country where the situation of prohibition is more pronounced, and new, strict norms of interaction with the environment are introduced, the increased level of anxiety and depression is more pronounced.

Введение. Пандемия COVID-19, начавшаяся в феврале 2020 года, внесла изменения в повседневную жизнь людей во всем мире, изменила ритм текущих процессов. Никто не мог предвидеть и не ожидал закрытия границ, ограничения выхода из дома, перевода работы и обучения в онлайн форматы. Тот факт, что данные ограничения повлияют на психику людей, не вызывал сомнения уже в начале пандемии, однако какие это будут изменения, и насколько глубоко они затронут людей, прогнозов никто дать не мог. Настоящее исследование направлено на определение уровней состояния личной тревожности и депрессии студентов.

Глобальный кризис здравоохранения, вызванный пандемией COVID-19 и характеризуемый высокими уровнями неопределенности, риска и угрозами для жизни, оказал сильное влияние на уровень тревожности людей. Высокий коэффициент распространения болезни, отсутствие методов гарантированного лечения и неопределенность всего процесса пандемии представляли особый риск для психического здоровья общества в целом. Кроме того, сокращение социальных контактов и практика карантина усилили тревогу и беспокойство людей. Мы стремились изучить влияние глобального кризиса, вызванного COVID-19, на психическое здоровье студентов, вынужденных перенести свое обучение в онлайн формат.

Целью исследования было сравнить тревожность и депрессивность у турецких и российских студентов в период второй волны пандемии 2020 года. Опросы студентов проводились в декабре 2020 года. Выбор стран был обусловлен разным государственным отношением к эпидемиологической обстановке осенью 2020 года. В Турции был введен довольно строгий режим самоизоляции, гражданам не разрешался свободный выход из домов, маски необходимо было носить на улицах, все обучение велось онлайн, спортивные клубы были закрыты. В России в этот период можно было гулять, передвигаться на улице без масок, заниматься спортом, обучение практиковалось в двух формах: онлайн и офлайн.

В процессе подготовки статьи мы рассматривали современные исследования, посвященные сходной тематике и опубликованные в турецких научных изданиях $[1 ; 11]$.

Литературный обзор проблемы. Тревога определяется 3. Фрейдом как «переживаемое, эмоциональное состояние, включающее в себя чувство опасения, напряжения, нервозности и беспокойства, сопровождающееся физиологическим возбуждением» [2]. Согласно определению Американской психиатрической ассоциации [3], тревога - это реакция тела человека на предполагаемую угрозу, характеризующуюся тревожными мыслями, напряжением, повышенным кровяным давлением, частотой дыхания, пульсом, потоотделением, затрудненным глотанием, головокружением или болью в груди, которая вызвана убеждениями, эмоциями и мыслями человека.

Базовые модели психопатологии, связанной с тревогой, указывают на то, что тревога и страх состоят из трех основных компонентов. Это физиологические (активация автономной нервной системы), когнитивные (интерпретация внешних и физиологических стимулов) и поведенческие (избирательная реакция на стимулы) реакции. Страх рассматривается как защитная реакция на угрожающую ситуацию с сильными физиологическими реакциями и поведением, направленным на борьбу или бегство от источника. Тревога физиологически схожа со страхом, потому что она усиливает автономное возбуждение. С другой стороны, тревога возникает в ответ не на существующую идентифицируемую угрозу, а в отношении потенциальной угрозы. Важным когнитивным отличием тревожности является чувство неконтролируемости, которое учитывает возможность будущих угроз, опасностей или других потенциальных негативных событий [4]. Страх и тревогу можно разделить с точки зрения того, насколько они уверены в вероятности, времени или качестве будущей угрозы [5].

Негативные жизненные события с потенциальными угрозами, такими как межличностный конфликт, болезнь, финансовые трудности, потеря, траур, часто вызывают тревожные расстройства [6]. В стрессовых ситуациях как жизненные кризисы и массовые 
травмы, тревожные паттерны наблюдаются не только у тех, кто подвергается прямому воздействию, но и косвенно, так же как, например, воздействию образов средств массовой информации [7]. Прогнозировалось, что восприятие угрозы и неопределенности в процессе пандемии COVID-19 вызовет проблемы c тревогой не только у больных, их родственников, медицинских и социальных работников в сфере общественного здравоохранения, но и у всего населения, подверженного этому восприятию.

На основе взаимодействия между человеком и окружающей средой процесс когнитивной оценки определяет, почему и насколько стрессовой является ситуация. Когда люди сталкиваются с жизненным кризисом, они, в первую очередь оценивают, важны ли потенциальные факторы стресса для благополучия их самих и людей, которых они любят. Когда событие воспринимается как угрожающее, запускается вторичный процесс оценки, в ходе которого возникают стрессовые реакции. Здесь люди оценивают психологические (самооценка, оптимизм, самоконтроль и т.д.), личные (доход, образование, профессиональный статус и т.д.) и социальные (устойчивость семьи, социальная поддержка и т.д.). Ресурсы, которые повышают устойчивость и способность справляться с трудностями [8;10].

Мы будем считать, что тревога - это неприятное эмоциональное состояние, которое проявляется в ощущении неопределенности, беды, напряжения, страха, которые испытывают живые существа. Депрессия - распространенное и серьезное, но поддающееся лечению медицинское заболевание, которое отрицательно влияет на то, как чувствует, как думает и ведет себя человек. Депрессия приводит к постоянному состоянию грусти и неспособности наслаждаться ситуациями, которые доставляют удовольствие. Депрессия может выражаться различными эмоциональными и физическими симптомами. И тревога, и депрессия сильно снижают качество жизни, ухудшают психическое и физическое состояние человека.

Методы исследования и исходные данные. В качестве методики исследования была выбрана госпитальная шкала тревоги и депрессии HADS (The Hospital Anxiety and Depression Scale), разработанная для первичного выявления депрессии и тревоги [9;11]. Данная шкала помогает понять эмоциональное состояние человека. Выбор методики был связан с тем, что она переведена на национальные языки России и Турции и соответствует всем психометрическим требованиям.

В исследовании приняли участием 50 студентов из России и 50 студентов из Турции; возраст испытуемых от 18 до 21 года.

Результать исследования. Клиническая выраженность депрессии проявляется у 7\% российских студентов и $33 \%$ турецких студентов. Субклиническая выраженность депрессии наблюдается у $13 \%$ российских студентов и $20 \%$ турецких студентов. Нормальная выраженность депрессии выявляется у $80 \%$ российских студентов и $47 \%$ турецких студентов. Результаты исследования выраженности депрессии представлены на рисунке 1.

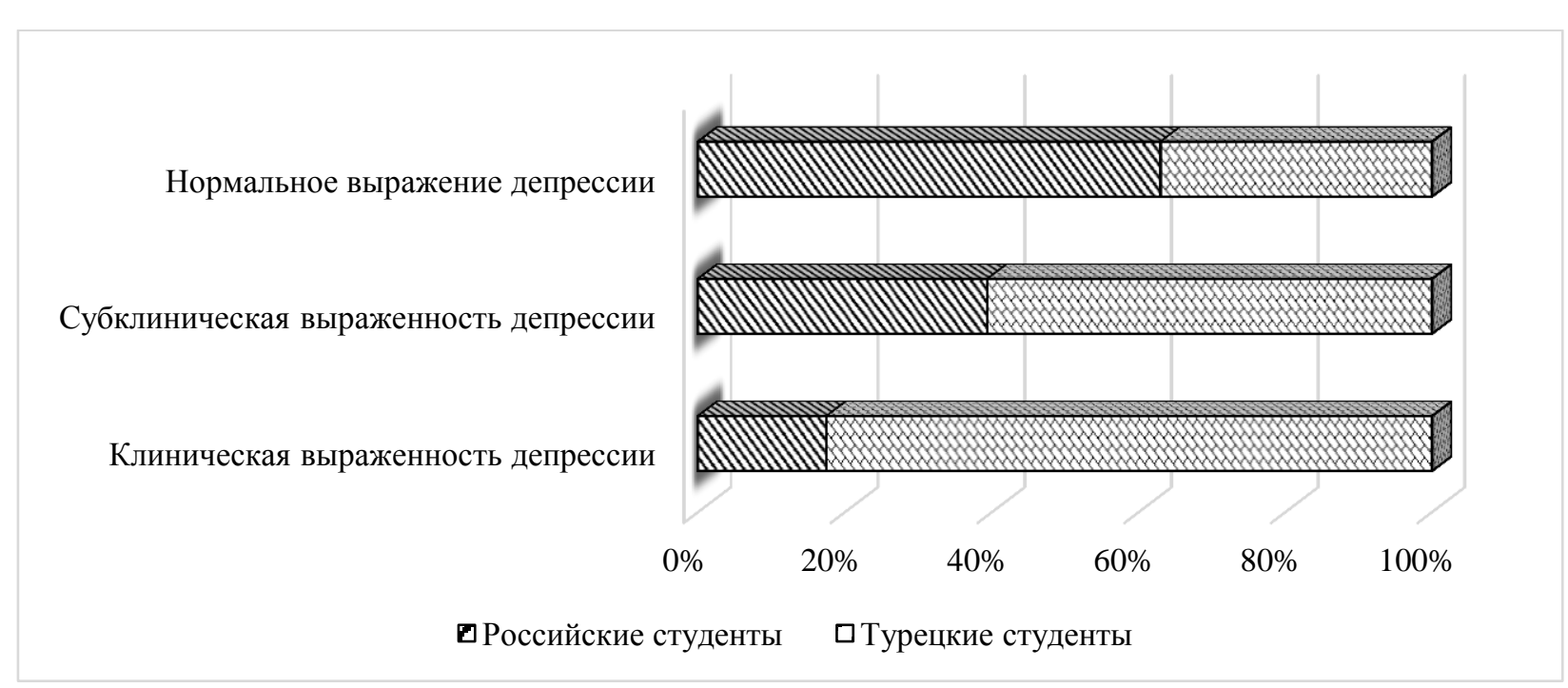

Рисунок 1. - Результаты исследования депрессии 
Клиническая выраженность тревоги у российских студентов составляет $27 \%$, у турецких - 73\%. Субклиническая выраженность тревоги выявляется у $20 \%$ как российских, так и турецких студентов. Нормальное выражение тревоги наблюдается у $53 \%$ российских студентов и у $7 \%$ турецких студентов. Результаты исследования выраженности тревоги представлены на рисунке 2.

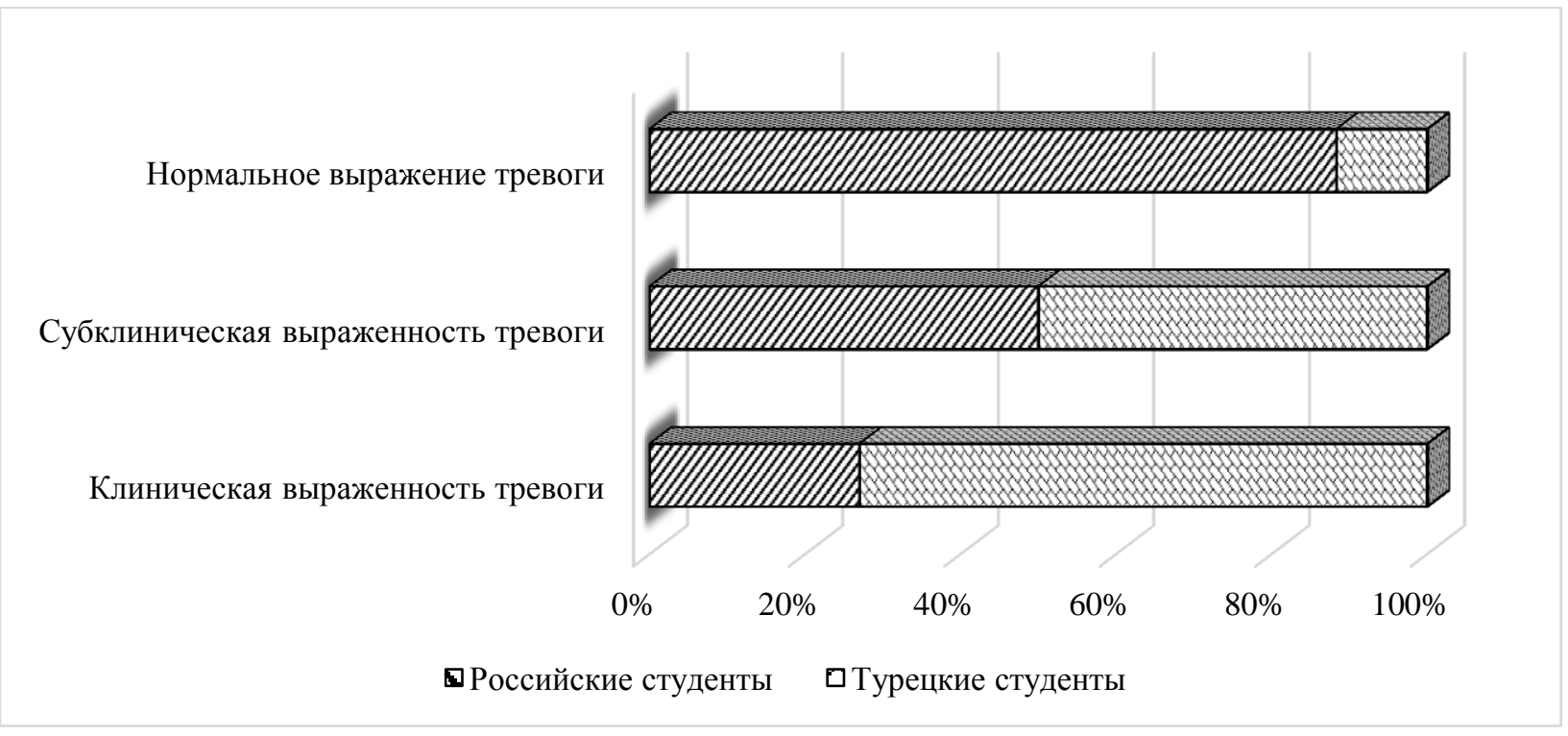

Рисунок 2. - Результаты исследования тревоги

Согласно полученным данным, мы можем сказать, что уровень тревожности и депрессии отличается в России и Турции. У российских студентов нормальное выражение в шкалах тревоги статистически значимо (p>0,5) выше, чем у турецких студентов. Также мы видим статистические значимые различия в показателях клинической выраженности депрессии $(\mathrm{p}>0,5)$.

Для турецких студентов процесс самоизоляции, в гораздо большей степени обострил проблемы тревоги и депрессии. Мы связываем это с более строгими государственными мерами по противодействию распространению коронавирусной инфекции.

Турецкие студенты большую часть своего времени вынуждены проводить в условиях замкнутого пространства, имеют ограниченные возможности прогулок, в том числе у них отсутствует свободный доступ к свежему воздуху, что усиливает психофизиологические симптомы тревоги и депрессии. Длительное нахождение в одной и той же обстановке, общение только с одними и теми же людьми (в основном с родителями), также повышает эмоциональное напряжение. Конфликты, ссоры людей, находящихся долгое время в замкнутом пространстве, снижают адаптивные возможности и повышают уровень стресса. Режим работы ресторанов и кафе с ноября сократился, они работают с 10 до 20 часов, продавая еду только на вынос. В то время как в России в ноябре рестораны и кафе были открыты для посетителей с 8 до 23 часов, работала большая часть залов, в которых гости могли проводить время за приемом пищи. Российские студенты имеют доступ в спортивные секции, могут встречаться со своими друзьями, посещать учебные заведения. Несмотря на продолжающиеся нормы по ограничению, в целом обстановка в России более свободная, чем в Турции, что и способствует невыраженному проявлению клинической и субклинической тревоги и депрессии.

Заключение. Пандемия COVID-19 особенно повысила уровень тревожности, которая усиливается в стрессовых условиях жизни. Исходя из проведённого исследования, мы видим, что в стране, где более выраженная ситуация запрета, и введены новые, строгие нормы взаимодействия с окружающей средой, более выражено проявляется повышенный уровень тревоги и депрессии.

Данное исследование позволяет по-новому взглянуть на ситуацию пандемии COVID-19, оценивая эмоциональное состояние людей, переживающих моменты изменения своего обычного ритма жизни. Объектом исследования стали эмоциональные состояния студенты России и Турции. Студенты являются именно той социальной группой населения, которая через 
несколько лет будет активным участником социальных и экономических отношений в своих государствах, а также на мировом пространстве. Авторы исследования, анализируя текущее эмоциональное состояние молодежи, делают вывод, что, вводя или отменяя меры по предотвращению распространения болезни, необходимо также учитывать возможный долгосрочный вред от данных мер. Отсроченная реакция на стресс может быть гораздо более серьезна, чем текущее эмоциональное состояние. Полученные данные позволяют говорить о необходимости рассмотрения возможности эмоциональной разрядки накопившейся негативной энергии в мирном, продуктивном русле. На данный момент мы видим, как в странах с усиленными мерами по предотвращению распространения COVID-19 возникают несанкционированные митинги, переходящие в вооруженные столкновения с полицией. Молодежь, в том числе и студенты университетов, устраивают драки, разбивают витрины, поджигают магазины, пытаясь справиться со своей тревогой и депрессией. Ни в одной стране пока не наблюдаются меры, помогающие справиться с данным состоянием. Более того, средства массовой информации продолжают нагнетать обстановку, пугая население третьей, а может быть и уже четвертой волной пандемии.

В эффективном разрешении данной ситуации активно могут участвовать, прежде всего, учебные заведения, проводя семинары, тренинги, встречи, в том числе и онлайн, позволяющие студентам говорить о своих страхах, переживаниях, делиться своими мыслями, анализируя свое эмоциональное состояние.

\section{Лumepamypa:}

1. Özmete E., Pak M. The relationship between anxiety levels and perceived social support during the pandemic of COVID-19 in Turkey // Social Work in Public Health. - 2020. - T. 35. - № 7. - C. 603-616.

2. Spielberger C.D., Edwards C.D., Lushene R.E., Montuori J., \& Platzek D. State-trait anxiety inventory for children: Preliminary manual // Consulting Psychologists, Palo Alto, CA. - 1973.

3. American Psychiatric Association A.P. et al. Diagnostic and statistical manual of mental disorders: DSM-5. - 2013.

4. Carleton R.N. The intolerance of uncertainty construct in the context of anxiety disorders: Theoretical and practical perspectives // Expert Review of Neurotherapeutics. - 2012. - T. 12. - № 8. - C. 937-947.

5. Rosen J.B., Schulkin J. From normal fear to pathological anxiety // Psychological review. - 1998. - T. 105. - № 2. - C. 325.

6. Panayiotou G., Karekla M. Perceived social support helps, but does not buffer the negative impact of anxiety disorders on quality of life and perceived stress // Social psychiatry and psychiatric epidemiology. - 2013. T. 48. - № 2. - C. 283-294.

7. Grills-Taquechel A.E., Littleton H.L., Axsom D. Social support, world assumptions, and exposure as predictors of anxiety and quality of life following a mass trauma // Journal of Anxiety Disorders. - 2011. - T. 25. № 4. - C. 498-506.

8. McCauley M., Minsky S., Viswanath K. The H1N1 pandemic: media frames, stigmatization and coping // BMC Public Health. - 2013. - T. 13. - № 1. - C. 1-16.
9. Zigmond A.S., Snaith R.P. The hospital anxiety and depression scale // Acta psychiatrica scandinavica. 1983. - T. 67. - № 6. - C. 361-370.

10. Tosun N. Distance education practices at universities in Turkey: a case study during covid-19 pandemic: Distance education practices // International Journal of Curriculum and Instruction. - 2021. - T. 13. - № 1. - C. 313-333.

11. Colaceci S. et al. Long-Term Effectiveness of an e-Learning Program in Improving Health Care Professionals' Attitudes and Practices on Breastfeeding: A 1-Year Follow-Up Study //Breastfeeding Medicine. - 2020. - T. 15. - № 4. - C. 254-260.

12. George P.P. et al. Online eLearning for undergraduates in health professions: a systematic review of the impact on knowledge, skills, attitudes and satisfaction // Journal of global health. - 2014. - T. 4. - № 1.

13. Leung P., Keing C. SARS hits in education: how we lived through it and what we have learned //Educational Research Journal. - 2003. - T. 18. - № 2. - C. 27-38.

14. Schleicher A. The impact of covid-19 on education insights from education at a glance 2020 // Retrieved from oecd.org website: https://www.oecd.org/education/the-impact-of-covid-19on-education-insights-education-at-a-glance-2020.pdf. 2020.

15. Tosun N. Distance education practices at universities in Turkey: a case study during covid-19 pandemic: Distance education practices // International Journal of Curriculum and Instruction. - 2021. - T. 13. - № 1. - C. 313-333.

\section{References:}

1. Özmete E., Pak M. The relationship between anxiety levels and perceived social support during the pandemic of COVID-19 in Turkey // Social Work in Public Health. - 2020. - T. 35. - № 7. - C. 603-616.
2. Spielberger C.D., Edwards C.D., Lushene R.E., Montuori J., \& Platzek D. State-trait anxiety inventory for children: Preliminary manual // Consulting Psychologists, Palo Alto, CA. - 1973 
3. American Psychiatric Association A.P. et al. Diagnostic and statistical manual of mental disorders: DSM-5. - 2013.

4. Carleton R.N. The intolerance of uncertainty construct in the context of anxiety disorders: Theoretical and practical perspectives // Expert Review of Neurotherapeutics. - 2012. - T. 12. - № 8. - C. 937-947.

5. Rosen J.B., Schulkin J. From normal fear to pathological anxiety // Psychological review. - 1998. - T. 105. - № 2. - C. 325.

6. Panayiotou G., Karekla M. Perceived social support helps, but does not buffer the negative impact of anxiety disorders on quality of life and perceived stress // Social psychiatry and psychiatric epidemiology. - 2013. T. 48. - № 2. - C. 283-294.

7. Grills-Taquechel A.E., Littleton H.L., Axsom D. Social support, world assumptions, and exposure as predictors of anxiety and quality of life following a mass trauma // Journal of Anxiety Disorders. - 2011. - T. 25. № 4. - C. 498-506.

8. McCauley M., Minsky S., Viswanath K. The H1N1 pandemic: media frames, stigmatization and coping // BMC Public Health. - 2013. - T. 13. - № 1. - C. 1-16.

9. Zigmond A.S., Snaith R.P. The hospital anxiety and depression scale // Acta psychiatrica scandinavica. 1983. - T. 67. - № 6. - C. 361-370.

10. Tosun N. Distance education practices at universities in Turkey: a case study during covid-19 pandemic: Distance education practices // International Journal of Curriculum and Instruction. - 2021. - T. 13. - № 1. - C. 313-333.

11. Colaceci S. et al. Long-Term Effectiveness of an e-Learning Program in Improving Health Care Professionals' Attitudes and Practices on Breastfeeding: A 1-Year Follow-Up Study //Breastfeeding Medicine. - 2020. - T. 15. - № 4. - C. 254-260.

12. George P.P. et al. Online eLearning for undergraduates in health professions: a systematic review of the impact on knowledge, skills, attitudes and satisfaction // Journal of global health. - 2014. - T. 4. - № 1.

13. Leung P., Keing C. SARS hits in education: how we lived through it and what we have learned //Educational Research Journal. - 2003. - T. 18. - № 2. - C. 27-38.

14. Schleicher A. The impact of covid-19 on education insights from education at a glance 2020 // Retrieved from oecd.org website: https://www.oecd.org/education/the-impact-of-covid-19on-education-insights-education-at-a-glance-2020.pdf. 2020 .

15. Tosun N. Distance education practices at universities in Turkey: a case study during covid-19 pandemic: Distance education practices // International Journal of Curriculum and Instruction. - 2021. - T. 13. - № 1. - C. 313-333.

19.00.07 - Педагогическая психология

\section{Сведения об авторах:}

Корецкая Ирина Александровна (г. Москва, Россия), кандидат исторических наук, доцент, доцент кафедры психологии, Российский экономический университет им. Г.В. Плеханова, e-mail: ikoretskay@mail.ru

Васякин Богдан Сергеевич (г. Москва, Россия), доцент, заведующей кафедрой психологии, Российский экономический университет им. Г.В. Плеханова, e-mail: vasyakin.bs@ rea.ru

Кая Ангелина Абдурахмановна (г. Москва, Россия), студентка 3 курса кафедры психологии, факультет менеджмента, Российский экономический университет им. Г.В. Плеханова, e-mail: alex2228@list.ru

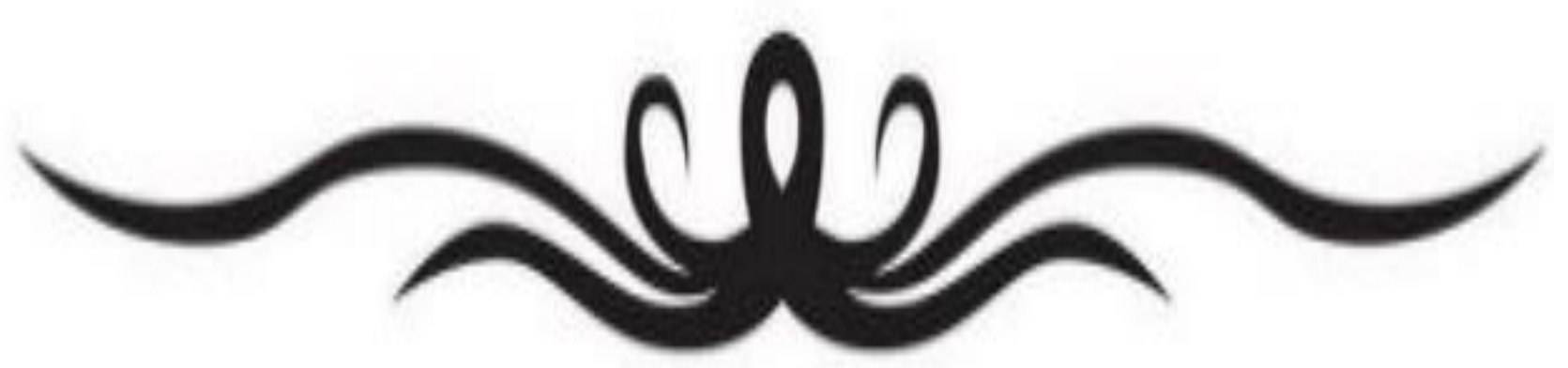

